\title{
Transferrin C Subtypes in Extremely Premature Newborn Infants
}

\author{
PIETRO AUCONI, ${ }^{(16)}$ RENZO BIAGINI, PATRIZIA COLARIZI, AND VINCENZO PASCALI \\ Pediatric Hospital, Arezzo, Italy [P.A., R. B.]; Third Department of Pediatrics, Università degli Studi, Roma, Italy \\ [P.C.]; and Department of Forensic Medicine, Università Cattolica, Policlinico "Gemelli," Roma, Italy [V.P.]
}

\begin{abstract}
Summary
Genetic typing of serum transferrin was performed in a group of 88 extremely premature infants (gestation age $<32 \mathrm{wk}$ ) and in a control group of 351 full-term infants, using isoelectric focusing technique on ultrathin layer of polyacrilamide gel. A major incidence of $\mathrm{C}_{2}$ type was found among the preterm infants when compared to full-term infants $\chi^{2}=22,86,(\mathrm{P}<0.001)$.

In view of the previously reported higher incidence of this phenotype in women prone to spontaneous abortion, a selective mechanism associated with this serum transferrin type promoting spontaneous abortion and preterm delivery, seems to occur. The relative risk of preterm delivery were calculated to 1.4 and 8.3 for the $C_{2-1}$ and $C_{2}$ types, respectively. Supportive evidence in favour of this hypothesis is offered by the correlation existing between transferrin $C_{2}$ allele and placental alkaline phosphatase variant $F$, the latter being associated with increased risk of spontaneous abortion.
\end{abstract}

\section{Speculation}

Assessment of the familial genetic pattern of serum transferrin may result to be a useful procedure for the evaluation of genetic risk of abortion and preterm delivery.

The molecular polymorphism of transferrin (Tf), the iron-binding $\beta$ globulin of the human serum, has been extensively studied by the use of starch gel electrophoresis (9); subsequently, the isoelectric focusing technique has brought evidence of a greater microheterogenity of the most commonly encountered $\mathrm{C}$ allele: two transferrin suballeles can be detected with this technique, $\mathrm{C}_{1}$ (anodal) and $\mathrm{C}_{2}$ (cathodal) $(7,13,14)$ (Fig. 1).

Recently, the Tf suballele $\mathrm{C}_{2}$ was found to have a high incidence in women prone to spontaneous abortion: the relative risk of abortion was found to be $2.3,1.4,0.6$ for the $\mathrm{C}_{2}, \mathrm{C}_{2-1}$, and $\mathrm{C}_{1}$ subtypes, respectively (2). The aim of the present study was to investigate on the relationships existing between the transferrin $\mathrm{C}$ polymorphism and extremely premature delivery.

\section{MATERIALS AND METHODS}

We have compared gene frequencies of the $\mathrm{Tf} C$ alleles in a group of 88 preterm infants (gestational age $<32 \mathrm{wk}$ ) and a group of 351 term controls taken at random from population of healthy newborn delivered in the same period of time. About $40 \%$ of the infants of these two groups originated from the province of Rome, the remaining $60 \%$ from the province of Arezzo (Tuscany). It was ascertained that gene frequencies of the $\mathrm{Tf}$ alleles are identical in the two provinces and therefore computation was operated regardless of the place of origin.

Cord serum samples were obtained at delivery and stored at $-20^{\circ} \mathrm{C}$ before the determination. The informed consent was obtained from the parents.
The gestational age was calculated on the basis of the date of the first day of the last menstrual period and on obstetric and neonatal objectivity (8). If discordance aroused between our estimation and that of the mother, the infant was excluded from the study.

Four preterm infants were deliberately excluded from this study because preterm delivery was ascribed to specific causes, such as uterus malformation, acute infection of the mother, age of the mother less than 14.

The genetic typing of serum transferrin was performed using isoelectric focusing technique, as described by Kueppers and Harpel (6), with minor modifications. With an ultrathin layer of polyacrylamide gel $(0.5 \mathrm{~mm}), 0.04 \mathrm{M}$ glutamic acid was used at the anode, $1 \mathrm{M}$ ethanolamine at the cathode and ampholytes in the pH range 4-6.5 (Pharmalytes, Pharmacia, Uppsala Sweden) were adopted, as previously described (9).

We noticed that preliminary treatment of the serum with half quantity of $13 \%$ 2-mercaptoethanol for $1 \mathrm{~h}$ allowed a sharper characterization of the $\mathrm{Tf}$ bands, without affecting the genetic pattern. The immunofixation technique described by Richie and Smiths (12) was employed.

The results were analyzed with the chi-square method. Rare Tf variants were omitted in the statistical analysis.

\section{RESULTS}

Table 1 summarizes the results concerning Tf gene frequencies of preterm infants and term controls. Similarly to what is observed in other European populations, $\mathrm{C}_{1}$ is the subtype most frequently encountered in the Italian population. Although the control sample agree with Hardy-Weinberg equilibrium, a distorsion exists of the phenotype distribution among the preterm newborn infants.

The frequency of the $C_{1}$ type is lower $\left(\chi^{2}=2.5957\right)$ in this group, whereas there is no difference concerning the $\mathrm{C}_{2-1}$ type $\left(\chi^{2}=0.2089\right)$. As a result of this distorsion of the phenotype distribution, the frequency of the $\mathrm{C}_{2}$ gene becomes higher among the preterm infants. The frequency of the $\mathrm{C}_{2}$ type is significantly higher $\left(\chi^{2}=22.86, \mathrm{P}<0.001\right)$ in the preterm infants when compared to full-term. From these data, the relative risk of preterm delivery can be estimated to be 1.4 and 8.3 for $\mathrm{C}_{2-1}$ and $\mathrm{C}_{2}$ subjects, respectively.

Thirty-one preterm infants had siblings who were delivered preterm (gestation age $<37 \mathrm{wk}$ ), or a maternal history of previous spontaneous abortion; twenty-four were affected by idiopathic respiratory distress syndrome. The frequency of the $C_{2}$ allele in these subgroups of preterm infants did not differ from the remaining preterms (data not shown).

We have also noticed that the preterm infants that developed idiopathic respiratory distress syndrome frequently exhibited lower serum Tf levels, when compared to preterm infants of the same gestational age; nevertheless, the genetic pattern of the serum transferrin was clearly detectable in these infants as well. 


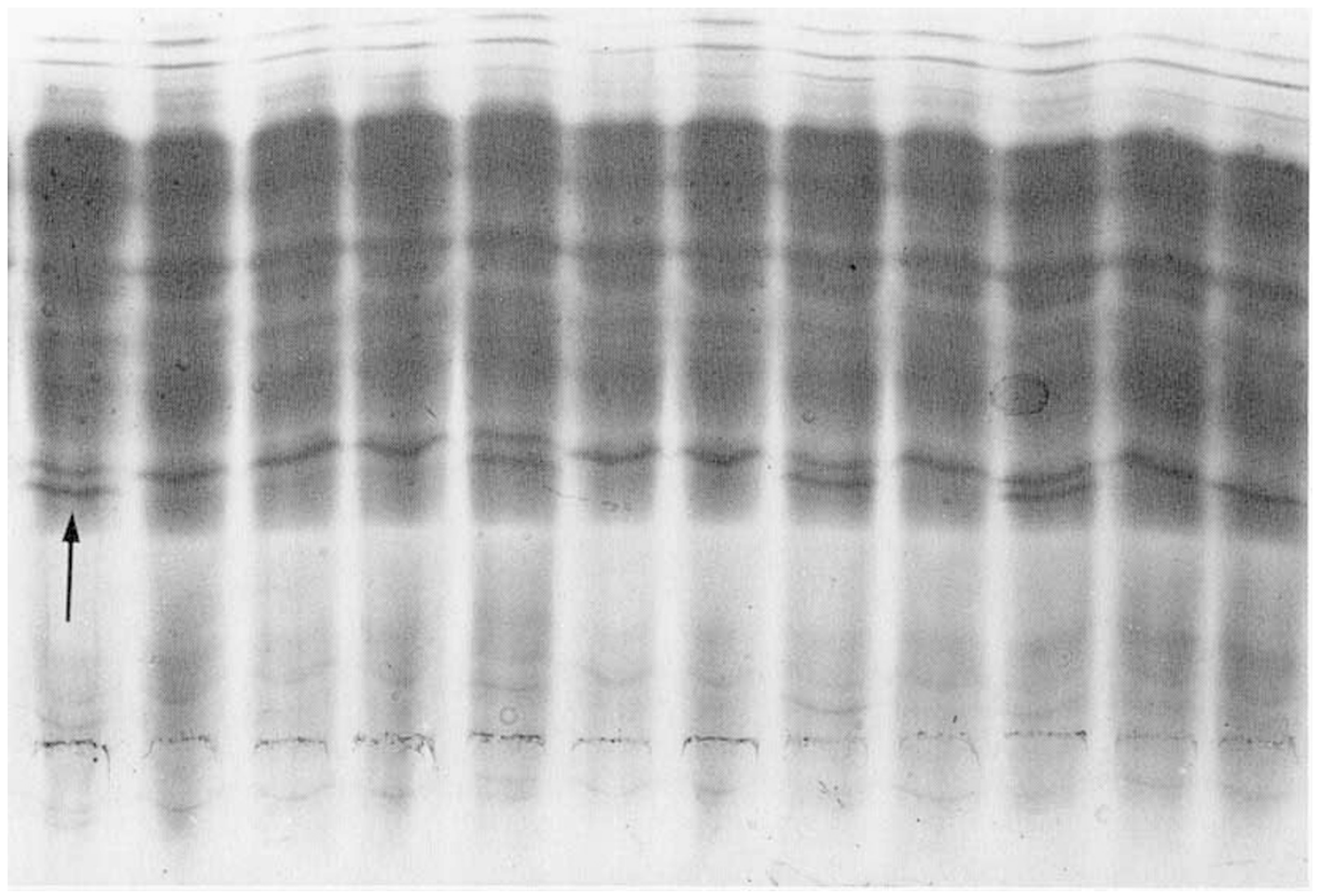

Fig. 1. Transferrin $\mathrm{C}$ subtypes after isoelectric focusing in the $\mathrm{pH}$ range 4-6.5. Anode is at the top. From left to right: $\mathrm{C}_{2-1}\left(\right.$ arrow), $\mathrm{C}_{1}, \mathrm{C}_{1}, \mathrm{C}_{1}, \mathrm{C}_{2-1}$, $C_{1}, C_{1}, C_{2-1}, C_{1}, C_{2-1}, C_{1}, C_{1}$

Table 1. Distribution of transferrin $C$ subtypes in preterm and fullterm newborn infants. Rare transferrin variants were omitted (in the preterm group: $I C_{1} D$; in the full-term group: $\left.3 C_{1} D, 2 C_{1} B\right)^{1}$

\begin{tabular}{lcc}
\hline & Preterm infants & Full-term infants \\
\hline Gestational age & $26-31$ & $37-42$ \\
Weight $(\mathrm{g})$ & $680-1750$ & $2310-4770$ \\
$\quad$ No. & 88 & 351 \\
$\mathrm{C}_{1}$ & & \\
$\quad$ observed & 46 & 237 \\
$\quad$ expected & 56.86 & 226.14 \\
$\mathrm{C}_{2-1}$ & & \\
$\quad$ observed & 28 & 101 \\
$\quad$ expected & 25.92 & 103.08 \\
$\mathrm{C}_{2}$ & & 8 \\
$\quad$ observed & 13 & 16.78 \\
$\quad$ expected & 4.22 & $\mathrm{pC}_{1}=0.8309$ \\
Gene frequencies & $\mathrm{pC}_{1}=0.6897$ & $\mathrm{pC}_{2}=0.1691$ \\
\hline
\end{tabular}

${ }^{1} \chi^{2}$ homogeneity population test $=25.6646$ and $\chi^{2} C_{2}$ type preterm infants $v s$ full-term infants $=22.86(P<0.001,2$ degrees of freedom $)$.

\section{DISCUSSION}

In many pathologic conditions, the influence of polygenic inheritance is presumed. When preterm delivery and abortion occur, the influence of individual and environmental factors is difficult to assess, but it can be reasonably assumed that genetic factors play an important role in these incidents $(3,5)$. We know that at least four polymorphic genetic systems, i.e., $\mathrm{ABO}, \mathrm{Rh}$, haptoglobin, placental alkaline phosphatase, may intervene simultaneously in the selection process that takes place during early intrauterine development and give rise to abortion, and possibly to preterm delivery and neonatal jaundice $(5,13)$. Interactions between these systems can produce pathologic effects in the fetus and in the newborn which are still largely unknown.

The results obtained by our study suggests that the Tf polymorphism contributes not only to spontaneous abortion, but also to preterm delivery: fetuses showing $C_{2}$ phenotype seem to have a higher risk of preterm delivery. The mechanism behind this phenomenon is completely unknown. Similarly obscure is the mechanism behind intrauterine selection mostly versus $\mathrm{C}_{2}$ omozygote fetuses, which leads to premature delivery, but it is unlikely to be caused by immunologic incompatibility between mother and fetus: a kind of disadvantage associated with, or caused by the $\mathrm{C}_{2}$ type, appears to be a plausible hypothesis at present.

It is remarkable that a correlation between $\mathrm{C}_{2}$ allele and placental variant $F$ of the alkaline phosphatase exists, the latter having been associated with spontaneous abortion (1).

Parker et al. (11), using starch gel electrophoresis reported additional faint cathodic components of the transferrin obtained from umbilical cord serum of preterm infants, probably caused by an incomplete sialylation of the molecule. Nonetheless, we have not observed such extrabands with the isoelectric focusing technique; moreover, we have accomplished $\mathrm{Tf}$ typing in both parents of eight preterm newborn infants and found that the modality of inheritance of the $\mathrm{Tf}$ alleles was autosomic codominant in every case examined, thus ruling out misinterpretations of cathodic extra bands caused by premature birth. It has been possible to repeat, after a few months, genetic typing of $\mathrm{Tf}$ in some infants belonging to the preterm group, using sera specimens collected for routine examinations. The second typing confirmed in every instance that the pattern found at birth was hereditary.

We believe these data demonstrate that $\mathrm{Tf}$ phenotype $\mathrm{C}_{2}$ is an important genetic factor related with preterm delivery, and suggest the usefulness of accomplishing genetic typing of $\mathrm{Tf}$ in both parents when a previous history of repeated spontaneous abortion or preterm delivery without known causes exists. Further investigations concerning assessment of the genetic pattern of transferrin on the parents are necessary to confirm these results, and to estimate the relative risk of abortion or preterm delivery, in view of the possible preventive implications.

\section{REFERENCES AND NOTES}

1. Beckman. G., Beckman, L., and Magnusson, S. S.: Placental alkaline phosphatase phenotypes and prenatal selection. Hum. Hered., 22: 473 (1972).

2. Beckman, G., Beckman, L., and Sikstrom, C.: Transferrin C subtypes and spontaneous abortion. Hum. Hered., 30: 316 (1980).

3. Bottini, E., Lucarelli, P., Pigram, P.. Palmarino, R., Spennati, G. F.. and Orzalesi 
M.: Interaction between placental alkaline phosphatase and $\mathrm{ABO}$ system polymorphisms during intrauterine life. Amer. J. Hum. Genet., 24: 495 (1972)

4. Bottini, E.: Abortion: a hipotesis on the role of $A B O$ blood groups and placental alkaline phosphatase. Soc. Biol., 22: 221 (1975).

5. Graven, S. N. and Misenheimer, H. R.: Respiratory distress syndrome and the high risk mother. Amer. J. Dis. Child.. 109: 489 (1965).

6. Kueppers, F. and Harpel, B.: Transferrin C subtypes in blacks and whites. Hum. Hered., 30: 376 (1980).

7. Kuhnl, P. and Spielman, W.: Transferrin. Evidence for two common subtypes of the Tf allele. Hum. Genet., 43: 91 (1978).

8. Lubcenko, L. O.: The high risk infant. pp. 9-64. (W.B. Saunders Co., Philadelphia. 1976).

9. Massi, G., Fabiano, A., Ragusa, D., and Auconi, P.: Characterization of Alphal-antitrypsin by isoelectric focusing on a ultrathin polyacrylamide gel layer. Hum. Genet., 53: 91 (1979)

10. Parker. W. C. and Bearn, A. G.: Additional genetic variation of human serum transferrin. Science, 137: 854 (1962).

11. Parker. W. C., Hagstrom. J. W. C., and Bearn A. G.: Additional studies on the transferrin of cord serum and cerebrospinal fluid. J. Exp. Med., 118: 975 (1963)

12. Ritchie, R. F. and Smith R.: Immunofixation. I. general principles and application to agarose electrophoresis. Clin. Chem., 22: 497 (1976).

13. Takano, K. and Miller J. R.: ABO incompatibility as a cause of spontaneous abortion: evidence from abortuses. J. Med. Genet., 9: 144 (1972).

14. Thymann, M.: Identification of a new serum protein polymorphism as transferrin Hum. Genet.. 43: 225 (1978).

15. The authors thank Professor E. Bottini for his criticism and suggestions during the preparation of this manuscript.

16. Requests for reprints should be addressed to: Dr. Pietro Auconi, Divisione Pediatrica Ospedale Arezzo, Via Curtatone 54, I 52100 Arezzo, Italy.

17. Received for publication December 28, 1981.

18. Accepted for publication March 10, 1982. 